\title{
PROPOSAL FOR A CAVITY POLARIMETER AT MIT-BATES
}

\author{
P. Cameron ${ }^{1}$, W. Barry ${ }^{3}$, M. Conte ${ }^{4}$, D.A.Goldberg ${ }^{3}$, K. Jacobs ${ }^{6}$, A. Luccio ${ }^{1}$,

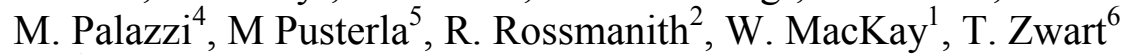 \\ ${ }^{1}$ Brookhaven National Laboratory, Upton, NY 11973, USA \\ ${ }^{2}$ Forschungszentrum Karlsruhe GmbH, D-76021 Karlsruhe, Germany \\ ${ }^{3}$ Lawrence Berkeley National Laboratory, Berkeley, CA 94720 USA \\ ${ }^{4}$ Universita and Sezione INFN di Genova, 16146 Genova, Italy \\ ${ }^{5}$ Universita and Sezione INFN di Padova, 35131 Padova, Italy \\ ${ }^{6}$ MIT-Bates Laboratory, Boston MA 01949 USA
}

\begin{abstract}
The possibility of successfully implementing a cavity polarimeter[1] has been greatly improved by the discovery[2] of a cavity mode for which the magnitude of the Stern-Gerlach force experienced by a magnetic moment traversing the cavity varies as the square of the relativistic factor gamma, so that the signal power varies as the fourth power of gamma. In addition, the interaction of this cavity mode with the beam charge varies as the inverse of the interaction with the magnetic moment, so that the background due to the beam charge varies as the inverse fourth power of gamma. If these gamma dependencies of moment and charge interaction with the pickup cavity do in fact exist, the possibility is opened for very fast, accurate, and inexpensive polarimetery at accelerators like MIT-Bates and RHIC. In addition, it might become possible to seriously consider SternGerlach polarization of beams at LHC. We present details of a quick polarimeter test at the electron storage ring at MIT-Bates, and of an extension of this test to a working polarimeter in the RHIC rings.
\end{abstract}

\section{POLARIMETER AT MIT-BATES}

The cavity polarimeter consists of a passive resonant cavity, which accumulates spin-dependent energy from the beam via the Stern-Gerlach interaction. In the case of a resonant cavity the stored energy increases until equilibrium is attained, where the power supplied by the beam equals the power dissipated as a result of the finite cavity Q. We can write this as

$$
Q \equiv \frac{\text { stored energy }}{\text { loss }}=\frac{\omega U}{\frac{d U}{d t}}
$$

where $\omega=2 \pi f, f=2.856 \mathrm{GHz}$ is the bunching frequency, and $\mathrm{U}$ is the stored energy. The rate at which polarization leaves energy in the cavity, or the signal power, is

$$
\frac{d U}{d t}=\mu B t b n f \gamma^{2} P
$$

where $\mu$ is the magnetic dipole moment of the electron, $\mathrm{B}$ is the cavity equilibrium field, $\tau \sim 0.7$ is the transit time factor, $b \sim 0.7$ is a bunch length factor, $n=10^{8}$ is the number of electrons per bunch for all buckets filled and 50ma beam current, $\gamma=2000$ is the relativistic gamma factor at

\footnotetext{
${ }^{*}$ Work performed under the auspices of the US Department of Energy.
}

$1 \mathrm{GeV}$, and $\mathrm{P} \sim 0.4$ is the beam polarization. Rearranging equation (1), we can write

$$
U=\frac{Q}{\omega} \frac{d U}{d t}=\frac{\varepsilon_{0}}{4} B^{2} c^{2} V
$$

Substituting (2) into (3) and solving for the equilibrium field B,

$$
B=\frac{4 Q \mu \tau b n \gamma^{2} P}{\pi \varepsilon_{0} V c^{2}}
$$

Where $\mathrm{Q}=5000, \varepsilon_{0}$ is the permittivity of free space, $\mathrm{c}$ is the speed of light, and $\mathrm{V} \sim 1000 \mathrm{~cm}^{3}$ is the cavity volume. Substituting the value found for the equilibrium field into (2), the signal power is

$$
{ }_{d t}^{\frac{1}{d U}}=\frac{4 Q f}{\pi \varepsilon_{0} V}\left(\frac{\mu \tau b n \gamma^{2} P}{c^{2}}\right)^{2} \approx 10^{-7} \text { watt }
$$

or about $-40 \mathrm{dBm}$. In the world of small signal beam instrumentation, this is a whopping big signal. In comparison, Schottky signals are typically at $-150 \mathrm{dBm}$ or less. It is interesting to note that the power varies as $\mathrm{f}^{4}$, because of the $1 / \mathrm{f}^{3}$ dependence in the cavity volume, so that there might be some advantage to operating the cavity at higher frequencies, and accepting the penalty in bunch length factor.

\section{POLARIMETER AT RHIC}

Performing the same calculation for conditions to be found at RHIC (55 bunches, $10^{11}$ per bunch, $50 \%$ polarization, $\gamma=266, \mathrm{Q}=5000$ ) yields the result that the signal power is about $-130 \mathrm{dBm}$. Again, this is a good strong signal relative to Schottky signals.

\section{CONCLUSION}

While a number of issues remain to be addressed, some airly straightforward and others thorny and difficult, the bvious course at this time is to build a simple cavity and perfom the measurement. We are proceeding with this as $\mathrm{r}^{\text {apidly as funding permits. }}$

\section{REFERENCES}

[1] Ya. S. Derbenev, "RF-resonance Beam Polarimeter: Part 1. Fundamental Concepts" NIM A 336, p.12-15 (1993).

[2] M. Conte et al, "The Stern-Gerlach Interaction between a travelling particle and a time varying magnetic field" http://xxx.lanl.gov/list/physics/0003, preprint 0003069 